\title{
Adjustment of cognitive control to the frequency of dual-task interference
}

\author{
Michal Olszanowski \\ SWPS University of Social Sciences \& Humanities in Warsaw, Department of Psychology

\section{Natalia Szostak} \\ SWPS University of Social Sciences \& Humanities in Warsaw, Faculty in Sopot, Department \\ of Psychology
}

\author{
Word count: 8500 \\ Abstract: 198 \\ Main text: 6800 \\ References: 49
}

Running head: Control adjustment in dual-tasking

Corresponding author: Michal Olszanowski, Psychology Department, University of Social Sciences \& Humanities in Warsaw, 19/31 Chodakowska Street, 03-815, Warsaw, Poland, e-mail: molszanowski@swps.edu.pl

Acknowledgements:

The authors would like to thank Bartosz Bednarz for his help with data acquisition. 
This study explored whether the control mechanisms recruited for optimizing performance are similar for dual-task and interference-task settings. We tested whether the frequency of appearance of a secondary task resulted in an adjustment of anticipatory and reflexive forms of attentional control, as has been observed with other interference tasks (e.g., stroop and flanker). The results of two experiments demonstrated a proportion congruency effect (PCE): when a secondary task frequently appeared, primary task performance was slower.

Additionally, there was a relative slowdown of dual-task performance in blocks wherein the secondary task appeared infrequently compared to blocks wherein it appeared frequently. However, this slowdown occurred when the primary task entailed a low level of control (Experiment 1) but was absent when it demanded a high level of control (Experiment 2). Overall, the results suggest that the level of control can be adjusted to task demands related to the frequency of the secondary task.

Keywords: Dual-task performance, Cognitive control, Response competition and interference, Control adjustment. 
In their everyday lives, people are frequently required to perform two tasks simultaneously (e.g. while driving a car there is a need to monitor both the position on the road and the speed). However, due to limitations of the human processing system, these tasks will compete for cognitive resources and thus performance on both tasks will be affected, causing the so-called dual-task interference (Pashler, 1994; Monsell, 2003). To optimize performance in such situations, cognitive control mechanisms, i.e. the processes which support flexible, adaptive and goal-directed responses and thoughts, are supposed to effectively manage the available cognitive resources to handle the tasks (McCann \& Johnston, 1992). In this paper, we present studies which investigate the connections between the cognitive control mechanisms involved in dual-task performance. More precisely, we sought to determine if the control mechanism adjusts to the frequency of dual-task interference, i.e. a situation wherein an additional (secondary) task appears while performing the first (primary) task.

The exact mechanism of dual-task coordination is still debated; however, there are two main theoretical accounts which are most often discussed. First, the so-called bottleneck account, suggests that except for stimulus perception and response execution, the processing of response selection required for both tasks needs to be performed in a serial manner and can be carried out on only one input at a time (e.g. Pashler, 1994). Therefore, cognitive control effectively manages the processing of stimuli through a bottleneck, with single tasks passing through consecutively (Lien, Ruthruff, \& Johnson, 2006). As a result, the processing of the second task is withheld until the processing of the first task is finished and the cognitive resources are capable of carrying out another task. An alternative account includes the central capacity-sharing models (e.g., Navon \& Miller, 2002; Tombu \& Jolicœur, 2003), which propose that processing capacity is shared between both tasks. According to this account, all stages of dual-task processing can be done in parallel, without the need for serial processing. Yet, when capacity is shared for response selection, performance on both tasks slows down. Despite their differences, both models assume that the presence of a secondary task results in between-task interference and a decrease in performance, which is either due to suspended processing of the second task, or limited resources which need to be shared between both tasks. Interestingly, recent research suggests that participants can process dual-tasks in either a serial (one task at a time) or a parallel (sharing capacity) manner (see Lehle, Steinhauser, \& Hübner, 2009; Orscheschek, Strobach, Schubert, \& Rickard, 2018; Strobach, Pashler, 
Schubert, \& Rickard, 2014). However, the underlying conditions of the adopted processing strategy remain unclear.

A better understanding of the control mechanisms involved in dual-task coordination might come from analyzing how cognitive control can deal with other types of interference. Aside from multi-task coordination, cognitive control is thought to play a role in basic mental functions (e.g. prioritizing or inhibiting behavior, providing resistance to information that is distracting etc.). As proposed by Miyake (Miyake et al., 2000), there are three executive functions: shifting between tasks and task settings, inhibiting prepotent responses, and updating of information, all of which contribute to cognitive performance to a different degree, depending on the task. We can further consider such functions in the context of a task traditionally thought to tap interference, e.g. Stroop. The task requires naming the color of the font (e.g., "blue", "green", or "red") in which a color word is printed. For incongruent trials, the color of the font, e.g., "blue", is different from the color denoted by the color-name, e.g., "red". In such a case, cognitive control can be understood as being involved in a competition between controlled (color-naming) and automatic (word-reading) processes. Here, the executive control mechanism needs to inhibit and override an erroneous automatic response, "red" when the correct response, the color in which the word is written is different: "blue," and thus a competitor.

An important idea in the literature related to cognitive control mechanisms is that the level of control can be adjusted to task demands (e.g. Carter et al., 2000; Logan \& Zbrodoff, 1979; West \& Baylis, 1998). It is assumed that the cognitive system continuously monitors for processing difficulties, and that their detection triggers compensatory processes to optimize performance which then can be adjusted both proactively (i.e. when predicting response interference) and retroactively (i.e. after noticing response interference via monitoring processes; Botvinick, Braver, Barch, Carter, \& Cohen, 2001; Braver, 2012). As a result, the level of attentional control can be voluntary when based on the anticipation of information, or reflexive when based on stimuli features (Bugg \& Crump, 2012; Schmidt, 2013). For example, when participants need to use cognitive resources to select appropriate responses to the stimuli, their levels of attentional control adapt to the frequency of incongruent trials . In this case, adaptation can be modulated e.g. by the relative proportion of incongruent/interference trials within an experimental block, resulting in the so-called proportion congruency effect (PCE; Logan \& Zbrodoff, 1979). Thus, when there is a high proportion of interference trials in the task (e.g. color-names written in different font color), 
attentional control is sustained even when non-interference trials appear (i.e. color-name and font color are identical). Consequently, non-interference responses are relatively extended and interference responses are relatively shortened, with the size of the effect varying with the proportion of interference trials - being larger in the context of a low proportion of interference trials than in the context of a high proportion of interference trials. In other words, it is assumed that there is a proactive (top-down regulated) control mechanism which can anticipate and prevent interference before it occurs by continuously and actively maintaining task-set information. Importantly, the mechanism of control adaptation has mainly been demonstrated in most of the classical interference and inhibition tasks such as: flanker, Simon, and Stroop (e.g. Abrahamse, Duthoo, Notebaert, \& Risko, 2013; Kuratomi \& Yoshizaki, 2016; Torres-Quesada, Funes, \& Lupiáñez, 2013). Here, we address a theoretically interesting question: is there similar flexibility in regulating dual-task performance? Or, to put it differently, can dual-task interference be regulated similarly to response interference in Stroop/flanker task, i.e. by adopting the optimal strategy of managing task-set information and task performance to maximize correctness with minimum resource-effort?

As a matter of fact, we can find theoretical and empirical rationale for such an expectation. First, we can refer to control theories of dual-tasking, such as the Executive Control Theory of Visual Attention (ECTVA; Logan \& Gordon, 2001). This theory specifies that when people are given explicit instructions for task performance, this can result in them setting and regulating their cognitive control in a top-down manner. Such a setting allows the cognitive system to protect itself against distraction from irrelevant stimulus attributes (i.e. task shielding) or switching between the tasks. Moreover, we can also observe voluntary regulation of cognitive control when performing a dual-task. After reviewing over 50 experimental reports, Fischer and Plessow (2015) concluded that adopting a parallel or a serial strategy of task processing changes with regards to the experimental conditions under which multiple tasks are performed. It means that processing of two tasks, A and B can be performed at the same time (i.e. according to predictions of the capacity sharing account) or one after another (i.e. as the bottleneck account predicts). For example, when not instructed to perform the task in any specific way, participants had a tendency to adopt a more parallel processing strategy, as compared to the more effortful serial processing, despite it causing an overall slowdown in dual-task performance (Lehle, Steinhauser, \& Hübner, 2009). However, when participants received instructions to perform dual-tasks in either a more parallel or a more serial fashion, greater levels of between-task interference were found when they adopted 
the parallel strategy. Importantly, switching between serial and parallel processing seems to be quite easy and participants can do it flexibly (Lehle \& Hübner, 2009). Other studies have shown that differentiating the stimulus onset asynchronies (SOAs) impacts the relative efficiency of the serial and parallel modes - with frequent short SOAs resulting in more parallel processing than frequent long SOAs (Miller, Ulrich, \& Rolke, 2009).

Apart from adopting a more serial or more parallel way of task processing, research also suggests a possibility of strategic allocation of processing resources and regulation of the response order. For example, in their recent work, Mittelstadt and Miller (2017) compared performance between two types of dual-task paradigms: psychological refractory period (PRP) and prioritized processing (PP). In the PRP paradigm, two tasks are presented sequentially and a participant should respond to them separately (Pashler, 1984). On the other hand, in the PP paradigm a participant is requested to respond mainly to the primary task (e.g. differentiating between specified two letters), but the response to the secondary task should only be executed if the primary task did not allow for a response (i.e. neither of the two “target” letters was shown - see Miller \& Durst, 2014). Mittelstadt and Miller (2017) compared the difference in responses to the primary task (i.e. letter differentiation) between trials in which a secondary task (i.e. discriminating the color of the square surrounding the letter) stimulus occurred and trials without any secondary task. As it transpired, the participants' reaction times were longer when the PRP paradigm was applied, as compared to the PP paradigm, despite the presence of absence of secondary task. The authors suggest that this was due to "preallocation" of processing resources, based on anticipated processing requirements. In other words, the PP paradigm required allocating more resources to process the primary task than the PRP, which resulted in more efficient responding to this task. Other research also suggests that control mechanism can be incorporated in task-order regulation, i.e. setting the proper sequence of responding to tasks (Kübler, Reimer, Strobach, \& Schubert, 2018). As it was shown, task-order control costs were higher when participants were instructed to keep the same order of responses in which the stimuli were presented, compared to free-order instructions. Kübler and colleagues (2018) conclude that this reflects global monitoring-based mechanisms which process and activate appropriate task-order control structures in working memory.

Studies have also shown that when multitasking, tasks can be ordered hierarchically. In an experiment by Hirsch, Nolden, and Koch (2017), three tasks were introduced that could be grouped into three different task pairs. The transition across the pairs was controlled, so 
that either the second task was always the same (e.g., T2-T1 vs. T3-T1) or the first task was always the same (e.g., T1-T2 vs. T1-T3). The results showed increased costs of a task-pair switch - the adaptation and response to the changed task setting was delayed compared to repeated task-pairs. This suggests that at a global level, cognitive control mechanisms can group dual-task settings together to create a higher-level single task, with participants forming a mental representation of both task sets. Such hierarchical representation has sustained activation, thus when the task pair changes, an update of both tasks is needed, despite the response setting for one of them being already available at the level of cognitive control (Hirsch, Nolden, Philipp, \& Koch, 2017). Interestingly, other studies have also shown performance costs being a consequence of a previous task requiring a response to either primary or both tasks (Olszanowski, Bajo, \& Szmalec, 2015). More precisely, a Gratton-like conflict adaptation was shown, as performance on a dual-task was faster when preceded by another dual-task, compared to a situation when it was preceded by a single primary task, whereas performance on a single primary task was slower when preceded by a dual-task, compared to a situation when two single primary tasks were presented consecutively. In a similar fashion, a Gratton-like effect occurred for sequential modulation of the so-called Backward Crosstalk Effect (BCE; Janczyk, 2016; Scherbaum, Gottschalk, Dshemuchadse \& Fischer, 2015). At the behavioral level, BCE is observed as a shorter response time to T1 when responses to both $\mathrm{T} 1$ and $\mathrm{T} 2$ are compatible in their spatial location (e.g. both require left hand and foot response; Hommel, 1998). In the aforementioned experiments, BCE was larger after compatible trials and reduced after incompatible trials.

The above-mentioned findings demonstrate that participants can in fact modify control parameters (e.g. processing strategy or task-shielding) while performing a dual-task and that such regulation can be strategic and executed in a top-down fashion. However, to this date, models of dual-task regulation did not address the possibility of cognitive control adjusting to the frequency of a secondary task. In other words, is there a regulated level of attentional control based on how often the secondary task appears with a series of primary tasks? It is important to note here that dual-task interference can be understood in two ways. The first and the most common meaning is that dual-task interference depends on the similarity of information between the tasks that need to be processed and thus greater similarity results in cross-talk interference (see Pashler, 1994). In such a case, stimuli information (e.g. stimuli in both tasks are conceptually similar) or response information (e.g. similar pattern of responses) is overlapping, and thus the occurring interference can be understood as an "outcome 
conflict" (Navon \& Miller, 1987). However, dual-task interference can also be understood as a competition of tasks for limited cognitive and attentional resources (Pashler, 1994). The aim of the presented studies is to provide support for strategic regulation of control settings to secondary task interference frequency, with "interference" understood as task competition. To this end, we asked participants to perform a primary task (T1) of color-naming (Experiment 1) or $n$-back (Experiment 2) that was supplemented with a secondary task (T2) of tone discrimination. Critically, the number of trials in which a secondary task appeared varied between blocks of trials, being either frequent or rare.

The basic hypothesis tested here assumes that the degree to which T2 processing affects $\mathrm{T} 1$ processing depends on the frequency of task competition that is experienced. More precisely, based on the sustained attentional control approach (Gratton, Coles, \& Donchin, 1992), we expect that in situations of high T2 frequency (i.e. when within a series of trials mainly dual-tasks are presented and there is frequent task interference/competition), participants will adopt a strategy based on an anticipatory and sustained form of attentional control, whereby goal-relevant information is highly activated (i.e. T2 response patterns) and the system is set to shield from interference (Carter et al., 2000; Logan \& Zbrodoff, 1979). Conversely, in a situation where $\mathrm{T} 2$ is less frequent, the participants would adjust to a low level of control, i.e. a late correction strategy whereby cognitive control processes are recruited to resolve interference only when needed, such as after the occurrence of T2. This should result in longer responses to $\mathrm{T} 1$ presented alone in blocks of frequent $\mathrm{T} 2$, compared to blocks where T2 occurs less frequently.

It was expected that the interference from dual-task competition due to a higher level of top-down control and sustained activation of response pattern (Logan \& Gordon, 2001) would be smaller in blocks of frequent T2. Thus, in dual-task situations (i.e. wherein both tasks are presented) responses to T1 should be faster in blocks of frequent T2, as control settings should allow for reducing the costs of task competition. Overall, we should observe a Gratton-like effect for T1 response pattern. Such a processing strategy should also be reflected in shorter $\mathrm{T} 2$ responses and inter-response intervals between $\mathrm{T} 1$ and $\mathrm{T} 2$ in blocks of frequent dual-tasking. Observing the control adjustment should also be possible based on the distribution of errors. In general, adjusting to a higher level of control should result in an overall lower number of errors in all types of task settings (e.g. T1-T2 and single T1) in blocks of a high frequency of $\mathrm{T} 2$.

\section{Experiment 1}


To test the above-mentioned predictions, we designed an experiment containing blocks of trials including either $20 \%$ or $80 \%$ of dual trials, so the secondary task interference could be frequent or infrequent. To eliminate the need for task shielding (and exclude it as a possible effect explanation), the participants were asked to perform tasks which required processing in different modalities - the primary task was a color-naming task, while the secondary task was a tone-discrimination task.

\section{Method}

Participants. Dual-task experiments usually test between 15-30 participants (e.g. Hirsch, Nolden, \& Koch, 2017; Lehle, Steinhauser, \& Hübner, 2009; Olszanowski, Szmalec, \& Bajo, 2015). Expecting that data from some participants might be excluded, we invited 33 undergraduate participants from the University of Social Sciences and Humanities in Warsaw, who participated for course credit. A post-hoc power analysis for repeated measures ANOVA and a sample varying between 30 and 33 participants was performed with G-Power software (Faul, Erdfelder, Lang, \& Buchner, 2007) and showed that the power varying between $80 \%$ and $95 \%$ (depending on corrections for data non-sphericity and correlation among repeated measures) can be expected when assuming a moderate effect size of $f=.3$ and $\alpha=.05$.

Apparatus and stimuli presentation settings. The participants were tested on a Pentium computer running Super-Lab 4.5 software and seated in front of a computer screen at a viewing distance of approximately $60 \mathrm{~cm}$. All stimuli were presented centrally on a 17-inch color monitor, and the size of the letter strings was 72 points. The tones lasted for $250 \mathrm{~ms}$ and were presented via earphones at two different frequencies: $300 \mathrm{~Hz}$ and $1100 \mathrm{~Hz}$. The participant responses were collected via Cedrus Reponse-Pad (RB-840). Two different sets of response mapping were designed (i.e. the primary task was attached to the index and middle fingers of either the left or the right hand - see Figure 1) and randomly selected for each participant.

Procedure. All participants performed the dual-task, where the primary task was to indicate the color (red or green) of a letter string (for all trials it was in "XXXXX" format). In the dual-task trials a tone was presented along with the letters, and the participants had to determine whether the tone was high or low.

The instructions highlighting the need for fast, yet as accurate as possible responses, and the priority of the color-naming task, were given on a computer screen at the beginning of the experiment, and between each block of trials. Each participant was presented with four experimental blocks containing 50 trials (200 in total). Two of the blocks were with a High 
Probability of Secondary Task (HiT2), consisting of $80 \%$ (i.e. 40) dual trials in which the secondary task appeared, while the other two blocks had a Low Probability of Secondary Task (LoT2), consisting of $20 \%$ (i.e. 10) dual trials. To avoid learning of contingent relationships between the letter color and tone appearance or its frequency (the so-called contingency learning effect, Schmidt 2013; 2014), blocks contained an equal number of trials linking all possible tone and color combinations, presented in a random order. The experimental design predicted two possible block presentation orders, alternating high and low proportions of secondary task appearance (i.e. ABAB or BABA). Sixteen training trials, including feedback about the expected responses, were administered before the first block to ensure that the instructions were understood. Blocks were separated with short, 30-second rest periods.

Each experimental trial was initiated by a fixation cross shown for $250 \mathrm{~ms}$. After that, the visual stimuli appeared and remained on the screen for $250 \mathrm{~ms}$. In case of dual-task trials, the stimuli were presented in parallel (i.e. SOA of $0 \mathrm{sec}$ ). Participants were given $3000 \mathrm{~ms}$ to respond, after which the next trial began (see Figure 1). A lack of response within this time was recorded as "no-response". There was a 2000 ms blank screen gap between trial presentation. Indications for possible answers were presented at the bottom of the screen after each trial. The response time was recorded relative to the onset of the stimulus.

Figure 1 here

\section{Results}

First, taking into account the speed of simple reaction time (Woods, Wyma, Yund, Herron \& Reed, 2015) we removed all responses below 300 ms (less than 1\%) as artifacts and then computed mean RTs for the correct responses to single- and dual-tasks (including correct order of responses T1-T2), as well as overall accuracy for each type of trial within the experiment. Non-responses were coded as errors and further analyzed with erroneous responses for accuracy. One participant's accuracy was low and consisted of $37 \%$ of errors, thus it was treated as an outlier and removed from further analyses. Responses from 32 participants were used in the below analyses. The data were analyzed with 2 × 2 repeated measures ANOVA, with the type of block (High Secondary Task Probability - HiT2 vs. Low Secondary Task Probability - LoT2) and the type of trial (SingleT1 vs. DualT1) as independent variables. 
Table 1 shows mean reaction times for all conditions. The analysis of RTs revealed a main effect of trial type, with T1 single trials being performed faster than T1 in dual-task, $F(1,31)=31.68, p<.001, \eta^{2}=.51$. The main effect of block - secondary task probability was not statistically significant, $F(1,31)=2.92, p=.098$. A two-way interaction between secondary task probability and the type of trial (SingleT1 vs. DualT1) was statistically significant, $F(1,31)=6.39, p<.05, \eta_{p}^{2}=.17$.

Paired samples t-tests showed that single T1 trials were performed faster when they appeared in LoT2 blocks than HiT2 blocks, $t(31)=-3.24, p<.01,95 \%$ CI [-89.96, -20.43]. The differences in $\mathrm{T} 1$ task responses during dual-task trials were not significantly different between LoT2 and HiT2 blocks, $t(31)=0.21, p=.832$. Additionally, in HiT2 blocks, single $\mathrm{T} 1$ trials were performed faster than when they appeared in dual-task situations (dual T1), $t(31)=-3.94, p<.001,95 \%$ CI $[-139.74,-44.37]$. The same was true for LoT2 blocks, $t(31)=$ $-5.86, p<.001,95 \%$ CI [-204.44, -98.91].

The responses to the $\mathrm{T} 2$ were significantly faster during HiT2 blocks than during LoT2 blocks, $t(31)=-5.86, p<.001,95 \%$ CI [-204.44, -98.91]. In LoT2 blocks, the difference between responses to the primary task (dual T1) and the secondary task (dual T2) was statistically significant, with faster responses occurring for the primary task, $t(31)=$ 8.14, $p<.001,95 \%$ CI [-323.09, -193.64]. The same trend was observed in HiT2 blocks, $t(31)$ $=-7.00, p<.001,95 \%$ CI $[-212.32,-116.55]$.

Table 1. Mean RTs in milliseconds (standard deviations) for study $1(N=32)$, for all conditions.

\begin{tabular}{lccc}
\hline & Single T1 & Dual T1 & Dual T2 \\
\hline LoT2 & $647(135)$ & $799(207)$ & $1057(238)$ \\
HiT2 & $702(153)$ & $794(182)$ & $959(192)$ \\
\hline
\end{tabular}

We also compared the inter-response interval (IRI), which was the additional time needed to respond to the secondary task after responding to the primary one. Here, we observed that frequent appearance of a dual-task shortened the interval: $M_{H i S e c}=164, S D=$ 133 vs. $M_{\text {LoSec }}=258, S D=180 ; t(31)=5.35, p<.001,95 \%$ CI $[66.7,148.7]$. 
Accuracy was high (the mean of error responses was below $4 \%$ - please see Table 2 for details) and there were no significant differences in error responses between blocks or trials.

Table 2. Error rates (standard deviations) for study $1(N=32)$, for all conditions.

\begin{tabular}{lccc}
\hline & Single T1 & Dual T1 & Dual T2 \\
\hline LoT2 & $.02(.02)$ & $.02(.03)$ & $.07(.06)$ \\
HiT2 & $.01(.02)$ & $.02(.02)$ & $.06(.07)$ \\
\hline
\end{tabular}

\section{Discussion}

The results of Experiment 1 confirmed that the frequency of secondary task appearance impacts the processing strategy within blocks of trials. In general, responses to T1 presented alone were slower in blocks of high frequency of T2 as compared to blocks where T2 occurred less frequently. The occurrence of $\mathrm{T} 2$ also slowed responses to $\mathrm{T} 1$, as compared to responses of $\mathrm{T} 1$ presented alone. However, against the predictions, T1 response times in dualtask trials did not differ between blocks of high and low frequency of dual-task situations. Importantly, frequent dual-tasking made responses to T2 faster as compared to rare dualtasking. This was also pronounced in shorter inter-response interval between primary and secondary tasks. Overall, the pattern of results reflects a Gratton-like proportion modulation and suggests that the degree to which primary processing is affected by a dual-task also depends on the frequency of the experienced task competition. More precisely, when the secondary task appears often, the adopted processing strategy is based on an anticipatory and sustained form of attentional control that is set to minimize the secondary task interference (Carter et al., 2000; Logan \& Zbrodoff, 1979).

It should also be noted that both tasks turned out to be relatively easy and the distribution of errors did not provide any conclusive data. This raises a question: If the processing of primary task response selection was not automated due to short practice, which factor reduced the engagement of attentional control in stimuli processing and response selection (Ruthruff, Van Selst, Johnston \& Remington, 2006)? Additionally, although the experimental procedure was designed to prevent the contingency learning effect (Schmidt, 2013 ; 2014), it should be noted that the results, at least partially, can be explained by the temporal learning account (Schmidt, Lemercier, \& De Houwer, 2014). The stimulus set 
consisted of only two types of stimuli for both T1 and T2. Given the relatively limited number of response combinations, the participants might have adopted a response strategy based on the predicted stimulus-response repetition. This, in turn, could have helped to automate the processing, reducing the dual-task interference and allowing for sped-up responding. Taking this into account, in Experiment 2 we wanted to increase task difficulty to rule out the possibility of contingency learning and automating response selection and increase the number of potential errors. This was achieved by introducing a task that continuously forces the engagement of cognitive resources, which would prevent participants from bypassing the processing of stimuli.

\section{Experiment 2}

The goal of Experiment 2 was to confirm the results from Experiment 1 by using a T1 that imposes a continuous cognitive load through which concurrent processing demands are maximized. Such a task setting would limit the possibility of automated task processing. We used an $n$-back updating task, which requires participants to decide whether each stimulus in a sequence matches the one that appeared $n$ items ago (e.g. Owen, McMillan, Laird, \& Bullmore, 2005). To correctly perform the $n$-back task, the participants have to hold and manipulate the information between the trials in working memory, hence the task strongly relies on executive control functions (Kane, Conway, Miura, \& Colflesh, 2007; Owen et al., 2005). As such, the participants are also required to maintain and update the information from trial to trial.

As in the previous experiment, we predicted that, in general, dual-task interference would be lower in the low-T2 probability condition than in the high-T2 probability condition. Moreover, we expected a generally higher level of errors committed by the participants performing the more demanding task, which would allow us to observe the impact of control engagement on accuracy.

\section{Method}

Participants. Based on the power achieved in the previous experiment and predicting some data exclusion, we tested 40 undergraduate students from the University of Social Sciences and Humanities in Warsaw, in exchange for course credit. Data from five participants were discarded, as they quit responding before the end of the experimental procedure (over $30 \%$ of trials were recorded as "no response"). As a result, responses from 35 participants were used in the below analyses. 


\section{Control adjustment in dual-tasking}

Apparatus and stimuli presentation settings. We used the same apparatus and stimuli settings as in Experiment 1.

Procedure. The experimental scenario was similar to that of Experiment 1 except for the primary task, which was a 1-back memory updating task with 18 randomly chosen letters from the Latin alphabet (i.e. A, B, C, E, G, H, J, K, L, N, O, P, R, S, T, W, Y, Z).

Additionally, each block consisted of 40 trials, two of which included a high proportion of secondary task trials (75\%, i.e. 30 trials where the secondary task appeared) and two including a low proportion of secondary task trials $(25 \%$, i.e. 10 trials where the secondary task appeared), resulting in a total of 160 trials. Half of the 1-back task trials were match trials (i.e. displayed letter matched the letter presented one position before). Again, to avoid the contingency learning effect (Schmidt 2013; 2014) the blocks contained an equal number of trials linking the possible letter and tone combinations, which were presented in a random order.

\section{Results}

As previously, the analyses were conducted on mean RTs for correct responses as well as error rates for the single- and dual-tasks. Responses below $300 \mathrm{~ms}$ (less than 1\%), as well as erroneous responses and non-responses, were removed for the analyses. The data were analyzed with $2 \times 2$ repeated measures ANOVA, with the type of block (HiT2 vs. LoT2) and the type of trial (SingleT1 vs. DualT1) as independent variables.

Table 3 shows mean reaction times for all conditions. The analysis of RTs showed a statistically significant main effect of trial type, $F(1,34)=188.99, p<.001, \eta^{2}=.85$. There was also a previously unobserved main effect of block type - the trials within LoT2 blocks were performed faster than within HiT2 blocks, $F(1,34)=23.13, p<.001, \eta^{2}=.41$. Nevertheless, as in Experiment 1, we found a statistically significant two-way interaction between secondary task probability and the type of trial, $F(1,34)=45.60, p<.001, \eta^{2}=.57$.

Paired samples t-tests revealed that single primary task trials were performed more quickly when appearing during LoT2 blocks than HiT2 blocks, which replicated the results from Experiment 1, $t(34)=-6.55, p<.001,95 \%$ CI [-200.32, -105.48]. There were no significant differences between LoT2 and HiT2 blocks in primary task responses during dualtask trials, $t(34)=-1.13, p=.267,95 \%$ CI [-54.04, 15.44]. Additionally, in HiT2 blocks, single primary task trials were performed faster than when they appeared in dual-task situations, $t(34)=-8.63, p<.001,95 \%$ CI $[-256.19,-158.56]$. The same was true for LoT2 blocks, $t(34)=-16.76, p<.001,95 \%$ CI $[-382.33,-299.61]$. 
The responses to the secondary task were not significantly faster during HiT2 blocks than during LoT2 blocks, $t(34)=1.67, p=.104,95 \%$ CI $[-7.75,79.06]$. In LoT2, the difference between responses to the primary task and the secondary task was statistically significant, with faster responses occurring for the primary task, $t(34)=-15.53, p<.001,95 \%$ CI [-392.55, -301.68]. The same was true for HiT2 blocks, $t(34)=-14.81, p<.001,95 \%$ CI [$332.25,-252.06]$.

Table 3. Mean RTs in milliseconds (standard deviations) for study $2(N=35)$, for all conditions.

\begin{tabular}{lccc}
\hline & Single T1 & Dual T1 & Dual T2 \\
\hline LoT2 & $826(189)$ & $1167(239)$ & $1514(213)$ \\
HiT2 & $978(251)$ & $1186(236)$ & $1478(201)$ \\
\hline
\end{tabular}

As in Experiment 1, we additionally compared the inter-response interval for the secondary task. The results showed that frequent appearance of a dual-task shortened the interval: $M_{H i S e c}=292, S D=117$ vs. $M_{\text {LoSec }}=347, S D=132, t(34)=3.97, p<.001,95 \% \mathrm{CI}$ $[26.8,83.12])$.

The mean of error responses was 12\%, higher than in Experiment 1 (see Table 4). The results showed statistically significant effects of trial, $F(1,34)=6.68, p<.05, \eta^{2}=.16$. However, the effects of block, $F(1,34)=2.26, p=.142, \eta^{2}=.06$, and the 2-way interaction, $F(1,34)=0.03, p=.872, \eta^{2}=.001$, were not statistically significant.

T-tests indicated that the only significant difference was found between SingleT1 and DualT1 in the LoT2 block, $t(34)=-2.93, p<.01,95 \%$ CI $[-0.08,-0.02]$, with less errors registered for single trials.

Table 4. Error rate (standard deviations) for study $2(N=35)$, for all conditions.

\begin{tabular}{lccc}
\hline & Single T1 & Dual T1 & Dual T2 \\
\hline LoT2 & $.08(.05)$ & $.13(.11)$ & $.19(.05)$ \\
HiT2 & $.09(.07)$ & $.14(.12)$ & $.09(.09)$ \\
\hline
\end{tabular}

\section{Discussion}


The aim of Experiment 2 was to confirm cognitive control adjustment to dual-task interference frequency for a situation wherein the primary task imposed a continuous load on working memory. This was undertaken to eliminate the possibility that the primary task was automated and helped to reduce dual-task interference in blocks of frequent T2 appearance. Similarly, as in Experiment 1, we observed that responses to T1 were significantly slower within blocks of frequent T2, which can be interpreted as a result of adopting a high control strategy. Also, for both frequency conditions, the appearance of T2 produced an overall slowdown in responding to T1. Similarly, there was no difference in response to T1 in a dualtask situation between blocks of different frequency of T2. In contrast to Experiment 1, there was no difference in the overall speed of responding to $\mathrm{T} 2$ between the experimental blocks, however, the inter-response interval was shorter in high T2 frequency blocks. Altogether, the pattern of results showed Gratton-like proportion modulation and support the notion that the adopted processing strategy is set to minimize the secondary task interference (Carter et al., 2000; Logan \& Zbrodoff, 1979).

Additionally, administration of a more control demanding primary task resulted in a relatively higher number of errors as compared to Experiment 1, whereas less control demanding task was administered. First, we can observe that the secondary task interfered with maintaining and updating the information in working memory and caused participants to commit more recognition errors during the 1-back task. Additionally, in blocks of rare secondary task appearance, there was a greater proportion of errors in that secondary task. This suggests that the need to frequently respond to a secondary task sustained the mental representations of the task set and thus mapping the correct response to presented stimuli was easier.

\section{General Discussion}

The aim of the presented studies was to provide support for strategic regulation of control settings to secondary task frequency. A question was posed about the nature of control adaptation, i.e. whether participants adopt a strategy based on an anticipatory and sustained form of control (Gratton, Coles, \& Donchin, 1992). The results support the assumption that the high frequency of a secondary task causes a higher level of cognitive control held between the trials to manage and regulate task processing and response selection. Such a control setting can be interpreted as a form of proactive and sustained attention that extended the processing time of single $\mathrm{T} 1$, but also allowed a reduction in processing time for a secondary task resulting in a shorter inter-response interval (Braver, 2012; Botvinick, Braver, Barch, 
Carter, \& Cohen, 2001). Additionally, in an instance of a less control demanding T1, high T2 frequency also reduced an overall response time of response in dual-task performance. In contrast, the infrequent presentation of a secondary task led to a low level of attentional control and cognitive control was mainly recruited when the secondary task occurred. As a result, single $\mathrm{T} 1$ was performed relatively fast, but the appearance of $\mathrm{T} 2$ decreased the performance accuracy. We can speculate that in such a case, adjusting the low level of attentional control resulted in a reactive control setting, wherein cognitive resources were recruited only when a secondary task appeared.

An alternative explanation of the observed effect could also be provided, in line with the high-order task organization account (Hirsch, Nolden, \& Koch, 2017). Based on this account, frequent T2 appearance would result in a hierarchical ordering of both tasks, allowing for faster dual-task processing. Additionally, responding to a single T1 would result in switching costs - i.e. relatively longer responses to a single T1. Correspondingly, rare T2 appearance would allow for fast single $\mathrm{T} 1$ processing but would result in switching costs when T2 appeared.

As can be observed, both approaches allow for similar expectations, but the hierarchical ordering strategy would additionally require switching between dissimilar response settings for T1-T2 and $\mathrm{T} 1$ presented on its own. This in turn should result in more errors in a single $\mathrm{T} 1$ when $\mathrm{T} 2$ is frequent. However, as far as the current data are concerned, it seems that adapting to a single $\mathrm{T} 1$ during frequent $\mathrm{T} 2$ does not entail switching costs related to accuracy of performance. On the other hand, we must note that among a series of experiments on hierarchical task processing published by Hirsch and colleagues (Hirsch, Nolden, \& Koch, 2017; Hirsch, Nolden, Philipp, \& Koch, 2017), there was only one that included a similar condition, i.e. adaptation to changes of only secondary task settings. In that study, the authors used the same T1 for all blocks of trials and presented T2 or T3 interchangeably as a secondary task (Experiment 2, Hirsch, Nolden, Philipp, \& Koch, 2017). Crucially, the observed switching costs for T1 between T1-T2 and T1-T3 pair transitions were pronounced in reaction times, but not in errors. Thus, the exact nature of control adjustment and processing strategy should be the matter of further investigation.

The comparison of performance on the primary task between single and dual-task conditions shows a relative slowdown of response. Considering how the data fit into theoretical accounts of dual-task processing, they provide additional support for the parallel processing strategy and the central capacity sharing model (Tombu \& Jolicœur, 2003). It can 
be noticed that the primary task response is delayed by secondary task appearance, which suggests that the tasks must share available processing capacity due to limited resources. Thus, there is an increase in the duration of processing during the period of shared capacity. Moreover, taking into the account an observation made by Mittelstadt \& Miller (2017), we can also speculate that frequent occurrence of a secondary task leads to the allocation of a greater amount of processing resources for this task. This, in turn, decreases resources allocated to the primary task and slows down its processing.

However, both the serial processing and the bottleneck approaches offer an explanation as to why the primary task response is delayed when multitasking. Pashler stated that: "Even though people can normally prepare two mappings at the same time, doing so nevertheless seems to have some cost, contributing additional slowing beyond that attributable to the central bottleneck" (Pashler, 1994, p. 231). If this is the case, then a need to map both $\mathrm{T} 1$ and $\mathrm{T} 2$ responses could result in a delayed $\mathrm{T} 1$ response selection. Alternatively, an EPIC (executive-process interactive control) model introduced by Meyer and Kieras (1997) assumes that response selection can be strategically delayed to prevent response reversals and/or confusion. Such deferment can be applied in particular when the time between the onset of one stimulus and another is short (short SOA) or where the stimulus and response are in the same modality. It must be mentioned here, however, that the original model rather refers to strategic deferment of T2 (but not T1). As an extension to this, in their recent work Pieczykolan and Huestegge (2019) posit the existence of a response-order control mechanism of action scheduling that adapts e.g. to particular task instructions. In the case of parallel presentation of stimuli for both tasks, a response to the task defined as primary can be a deferment to schedule responses in the right order and prevent reversal.

Importantly, as we already mentioned in the introduction section, participants can flexibly adopt a parallel or serial processing strategy depending on task requirements (Lehle, Steinhauser, \& Hübner, 2009; Orscheschek, Strobach, Schubert, \& Rickard, 2018; Strobach, Pashler, Schubert, \& Rickard, 2014). In line with previous observations of Lehle and Hübner (2009), current research suggests that a parallel strategy is preferred over a serial strategy, even if it leads to higher costs of primary task performance.

As we concluded, the pattern of results reflects control adjustment to interference frequency and resembles a proportion congruency effect (PCE) (Gratton, Coles, \& Donchin, 1992; Logan \& Zbrodoff, 1979). Usually, PCE emerges as a consequence of the relative proportion of interference trials in conventional inhibition tasks like Stroop or flanker. 
However, some observed differences must be mentioned here. Stroop-like interference can be understood as a competition between an automatic and a controlled process (e.g. executive control needs to inhibit automatic activation). Adjusting control is then expressed as efficiency of inhibiting an incorrect response. In contrast, dual-task coordination requires the control processes to proceed with bottleneck limitations and efficiently manage the available processing capacity. As described by Miyake and colleagues (2000), multi-task coordination is somewhat separable from cognitive functions, such as shifting, updating, or inhibiting. Nevertheless, we can assume that in both cases the main role of cognitive control is the execution of a correct response, and that adjusting the higher level of control improves overall task performance. Here it should be noted that slight improvement in dual-task performance was detected only in Experiment 1, while execution of the response to primary task in the dual-task situation was similar regardless of dual-task frequency in both experiments.

Although the experimental procedure was designed to prevent the contingency learning effect (Schmidt, 2013; 2014) it should be kept in mind for future investigations that, the current results can be explained, at least partially, by the temporal learning account (Schmidt, Lemercier, \& De Houwer, 2014). In Experiment 1, the stimulus set consisted of only two types of stimuli for both $\mathrm{T} 1$ and $\mathrm{T} 2$. Given the relatively small number of stimuli, the participants might have adopted a response strategy based on the predicted stimulusresponse repetition which, in turn, allowed for bottleneck bypassing and sped-up responding. The $n$-back task used in Experiment 2 was applied to rule out such a possibility, however, it would seem reasonable to also increase the number of items in stimulus sets in future studies. Likewise, Experiments 1 and 2 used different stimulus modalities to avoid task shielding. On the other hand, responses to both tasks were manual and one could note that the same response modality could also result in backward crosstalk and result in spatial compatibility effects (see, e.g., Janczyk, Pfister, Hommel, \& Kunde, 2014; Lien \& Proctor, 2000). Thus, further development of the current study should consider different modalities of responding.

\section{Conclusion}

Despite the aforementioned limitations, Experiments 1 and 2 provided additional evidence for adjustment of cognitive control to tasks varying in cognitive processing demands. The presented findings are in line with previous studies reporting control adaptation with regards to: serial vs. parallel processing strategy (Lehle, Steinhauser, \& Hübner, 2009), the need for task shielding (Fischer, Gottschalk, \& Dreisbach, 2014), conflict adaptation (Olszanowski, Bajo \& Szmalec, 2015), hierarchical task organization (Hirsch, Nolden, \& 
Koch, 2017), or task-order regulation (Kübler, Reimer, Strobach, \& Schubert, 2018). Despite the functional differences in response competition observed between interference tasks (e.g. Stroop or flanker task and dual-tasks), the observed pattern of results in our experiments suggests that response competition between interference tasks might be at least partially controlled by the same control loop. This is important, since it supports the view that the control mechanism can be an adaptive tool, allowing people to deal with various forms of tasks taking place in everyday situations.

\section{References:}

Abrahamse, E. L., Duthoo, W., Notebaert, W., \& Risko, E. F. (2013). Attention modulation by proportion congruency: the asymmetrical list shifting effect. Journal of Experimental Psychology: Learning, Memory, and Cognition, 39(5), 1552-1562.

Botvinick, M., Braver, T., Barch, D. Carter, C. \& Cohen, J. (2001). Conflict monitoring and cognitive control. Psychological Review, 108 (3), 624-652.

Braver, T. S. (2012). The variable nature of cognitive control: a dual mechanisms framework. Trends in cognitive sciences, 16(2), 106-113.

Bugg, J. M., \& Crump, M. J. (2012). In support of a distinction between voluntary and stimulus-driven control: A review of the literature on proportion congruent effects. Frontiers in psychology, 3, 367.

Carter, C. S., Macdonald, A. M., Botvinick, M., Ross, L. L., Stenger, V. A., Noll, D., \& Cohen, J. D. (2000). Parsing executive processes: strategic vs. evaluative functions of the anterior cingulate cortex. Proceedings of the National Academy of Sciences, 97(4), 1944-1948.

Faul, F., Erdfelder, E., Lang, A. G., \& Buchner, A. (2007). G* Power 3: A flexible statistical power analysis program for the social, behavioral, and biomedical sciences. Behavior research methods, 39(2), 175-191.

Fischer, R., Gottschalk, C., \& Dreisbach, G. (2014). Context-sensitive adjustment of cognitive control in dual-task performance. Journal of Experimental Psychology: Learning, Memory, and Cognition, 40(2), 399.

Fischer, R., \& Plessow, F. (2015). Efficient multitasking: parallel versus serial processing of multiple tasks. Frontiers in psychology, 6, 1366. 
Gratton, G., Coles, M. G. H., \& Donchin, E. (1992). Optimizing the use of information: Strategic control of activation of responses. Journal of Experimental Psychology: General, 121, 480-506. doi:10.1037/0096-3445.121.4.480

Hirsch, P., Nolden, S., \& Koch, I. (2017). Higher-order cognitive control in dual tasks: Evidence from task-pair switching. Journal of Experimental Psychology: Human Perception and Performance, 43(3), 569-580.

Hirsch, P., Nolden, S., Philipp, A. M., \& Koch, I. (2017). Hierarchical task organization in dual tasks: evidence for higher level task representations. Psychological research, 1-12.

Hommel, B. (1998). Automatic stimulus-response translation in dual-task performance. Journal of Experimental Psychology: Human Perception and Performance, 24(5), 1368.

Janczyk, M. (2016). Sequential modulation of backward crosstalk and task-shielding in dualtasking. Journal of experimental psychology: human perception and performance, 42(5), 631.

Janczyk, M., Pfister, R., Hommel, B., \& Kunde, W. (2014). Who is talking in backward crosstalk? Disentangling response-from goal-conflict in dual-task performance. Cognition, 132(1), 30-43.

Kane, M. J., Conway, A. R., Miura, T. K., \& Colflesh, G. J. (2007). Working memory, attention control, and the N-back task: a question of construct validity. Journal of Experimental Psychology: Learning, Memory, and Cognition, 33(3), 615.

Kübler, S., Reimer, C. B., Strobach, T., \& Schubert, T. (2018). The impact of free-order and sequential-order instructions on task-order regulation in dual tasks. Psychological research, 82(1), 40-53.

Kuratomi, K., \& Yoshizaki, K. (2016): Flexible adjustments of visual selectivity in a Flanker task, Journal of Cognitive Psychology, 28 (4), 462-473.

Lehle, C., \& Hübner, R. (2009). Strategic capacity sharing between two tasks: Evidence from tasks with the same and with different task sets. Psychological Research, 73(5), 707.

Lehle, C., Steinhauser, M., \& Hübner, R. (2009). Serial or parallel processing in dual tasks: What is more effortful?. Psychophysiology, 46(3), 502-509.

Lien, M. C., \& Proctor, R. W. (2000). Multiple spatial correspondence effects on dual-task performance. Journal of Experimental Psychology: Human Perception and Performance, 26(4), 1260. 
Lien, M. C., Ruthruff, E., \& Johnston, J. C. (2006). Attentional limitations in doing two tasks at once: The search for exceptions. Current Directions in Psychological Science, 15(2), 89-93.

Logan, G. D., \& Gordon, R. D. (2001). Executive control of visual attention in dual-task situations. Psychological Review, 108(2), 393.

Logan, G. D., \& Zbrodoff, N. J. (1979). When it helps to be misled: Facilitative effects of increasing the frequency of conflicting stimuli in a Stroop-like task. Memory \& Cognition, 7(3), 166-174.

Maquestiaux, F., Ruthruff, E., Defer, A., \& Ibrahime, S. (2017). Dual-task automatization: The key role of sensory-motor modality compatibility. Attention, Perception, \& Psychophysics, 80(3), 752-772.

McCann, R.S., \& Johnston, J.C. (1992) Locus of the single-channel bottleneck in dual-task interference. Journal of Experimental Psychology: Human Perception and Performance, 18(2), 471-484.

Meyer, D. E., \& Kieras, D. E. (1997). A computational theory of executive cognitive processes and multiple-task performance: Part I. Basic mechanisms. Psychological review, 104(1), 3 .

Miller, J., \& Durst, M. (2014). "Just do it when you get a chance": the effects of a background task on primary task performance. Attention, Perception, \& Psychophysics, 76(8), 25602574.

Miller, J., Ulrich, R., \& Rolke, B. (2009). On the optimality of serial and parallel processing in the psychological refractory period paradigm: Effects of the distribution of stimulus onset asynchronies. Cognitive psychology, 58(3), 273-310.

Mittelstädt, V., \& Miller, J. (2017). Separating limits on preparation versus online processing in multitasking paradigms: Evidence for resource models. Journal of Experimental Psychology: Human Perception and Performance, 43(1), 89.

Miyake, A., Friedman, N. P., Emerson, M. J., Witzki, A. H., Howerter, A., \& Wager, T. D. (2000). The unity and diversity of executive functions and their contributions to complex "frontal lobe" tasks: A latent variable analysis. Cognitive psychology, 41(1), 49-100.

Monsell, S. (2003). Task switching. Trends in cognitive sciences, 7(3), 134-140.

Navon, D., \& Miller, J. O. (1987). Role of outcome conflict in dual-task interference. Journal of Experimental Psychology: Human Perception and Performance, 13, 438-448. 
Navon, D., \& Miller, J. (2002). Queuing or sharing? A critical evaluation of the singlebottleneck notion. Cognitive psychology, 44(3), 193-251.

Olszanowski, M., Bajo, M. T., \& Szmalec, A. (2015). A conflict monitoring account of the control mechanisms involved in dual-tasking. Journal of Cognitive Psychology, 27(6), 704-714.

Orscheschek, F., Strobach, T., Schubert, T., \& Rickard, T. (2018). Two retrievals from a single cue: A bottleneck persists across episodic and semantic memory. Quarterly Journal of Experimental Psychology, 1747021818776818.

Owen, A. M., McMillan, K. M., Laird, A. R., \& Bullmore, E. (2005). N-back working memory paradigm: A meta-analysis of normative functional neuroimaging studies. Human Brain Mapping, 25(1), 46-59.

Pashler, H. (1994). Dual-Task Interference in Simple Tasks: Data and Theory. Psychological Bulletin, 116(2), 220-244.

Pieczykolan, A., \& Huestegge, L. (2019). Action scheduling in multitasking: A multi-phase framework of response-order control. Attention, Perception, \& Psychophysics, 1-24.

Ruthruff, E., Van Selst, M., Johnston, J. C., \& Remington, R. (2006). How does practice reduce dual-task interference: Integration, automatization, or just stageshortening?. Psychological research, 70(2), 125-142.

Scherbaum, S., Gottschalk, C., Dshemuchadse, M., \& Fischer, R. (2015). Action dynamics in multitasking: the impact of additional task factors on the execution of the prioritized motor movement. Frontiers in psychology, 6, 934.

Schmidt, J. R. (2013). Questioning conflict adaptation: proportion congruent and Gratton effects reconsidered. Psychonomic Bulletin \& Review, 20(4), 615-630.

Schmidt, J. R. (2014). Contingencies and attentional capture: the importance of matching stimulus informativeness in the item-specific proportion congruent task. Frontiers in psychology, 5, 540.

Schmidt, J. R., Lemercier, C., \& De Houwer, J. (2014). Context-specific temporal learning with non-conflict stimuli: proof-of-principle for a learning account of context-specific proportion congruent effects. Frontiers in psychology, 5, 1241.

Strobach, T., Schubert, T., Pashler, H., \& Rickard, T. (2014). The specificity of learned parallelism in dual-memory retrieval. Memory \& cognition, 42(4), 552-569. 
Tombu, M., \& Jolicœur, P. (2003). A central capacity sharing model of dual-task performance. Journal of Experimental Psychology: Human Perception and Performance, 29(1), 3-18.

Torres-Quesada, M., Funes, M. J., \& Lupiáñez, J. (2013). Dissociating proportion congruent and conflict adaptation effects in a Simon-Stroop procedure. Acta Psychologica, 142(2), 203-210.

Van Selst, M., Ruthruff, E., \& Johnston, J. C. (1999). Can practice eliminate the psychological refractory period effect? Journal of Experimental Psychology: Human Perception and Performance, 25(5), 1268.

West, R., \& Baylis, G. C. (1998). Effects of increased response dominance and contextual disintegration on the Stroop interference effect in older adults. Psychology and Aging, 13(2), 206-217.

Woods, D. L., Wyma, J. M., Yund, E. W., Herron, T. J., \& Reed, B. (2015). Factors influencing the latency of simple reaction time. Frontiers in human neuroscience, 9 , 131. 


\section{FIGURES}

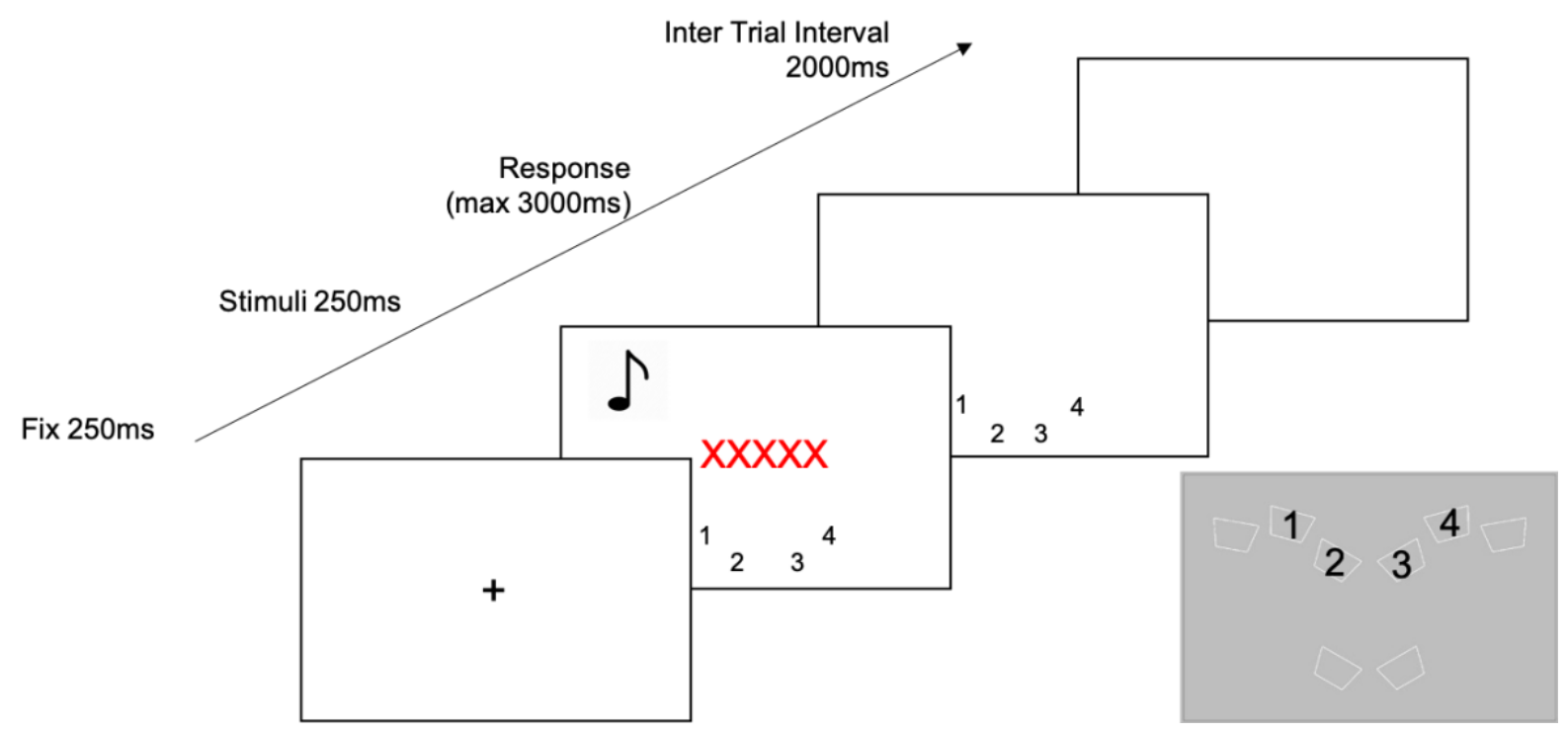

Figure 1. Trial schema for Experiment 1. Participant responses were collected via a Response Pad RB-840 (Cedrus). The keys at locations 1, 2, $3 \& 4$ were used during experimental procedure. Participants were asked to use their index and middle fingers of the left $\&$ the right hands. Half of the participants completed the test using keys $1 \& 2$ to respond to the primary task, while the other half used it for the secondary task. 